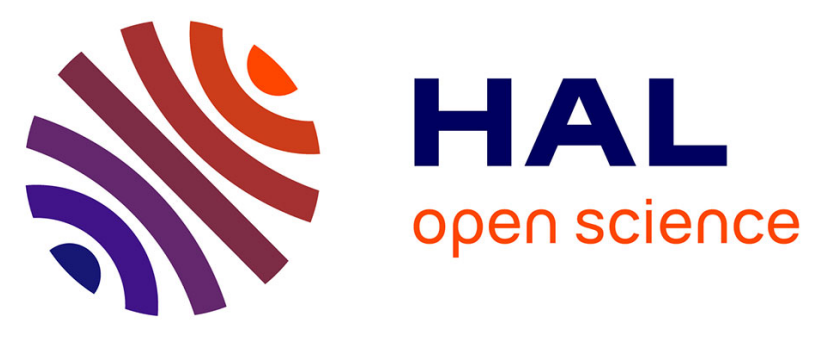

\title{
Millimetre-Wave Interrogation of Passive Sensors Embedded Inside Closed Reverberant Environments from Dual-Polarized Passive Repeaters
}

Timothée Marchal, Julien Philippe, Dominique Henry, Maria Valeria de Paolis, Anthony Coustou, Patrick Pons, Hervé Aubert

\section{To cite this version:}

Timothée Marchal, Julien Philippe, Dominique Henry, Maria Valeria de Paolis, Anthony Coustou, et al. Millimetre-Wave Interrogation of Passive Sensors Embedded Inside Closed Reverberant Environments from Dual-Polarized Passive Repeaters. 2019 49th European Microwave Conference (EuMC), Oct 2019, Paris, France. pp.33-36, 10.23919/eumc.2019.8910899 . hal-02381616

\author{
HAL Id: hal-02381616 \\ https://hal.laas.fr/hal-02381616
}

Submitted on 26 Nov 2019

HAL is a multi-disciplinary open access archive for the deposit and dissemination of scientific research documents, whether they are published or not. The documents may come from teaching and research institutions in France or abroad, or from public or private research centers.
L'archive ouverte pluridisciplinaire HAL, est destinée au dépôt et à la diffusion de documents scientifiques de niveau recherche, publiés ou non, émanant des établissements d'enseignement et de recherche français ou étrangers, des laboratoires publics ou privés. 


\title{
Millimetre-Wave Interrogation of Passive Sensors Embedded Inside Closed Reverberant Environments from Dual-Polarized Passive Repeaters
}

\author{
T. Marchal, J. Philippe, D. Henry, M. V. De Paolis, A. Coustou, P. Pons, H. Aubert \\ LAAS-CNRS, 7 avenue du Colonel Roche, F-31400 Toulouse, France \\ \{ tmarchal, jphilipp, dhenry, maria-valeria.de-paolis, acoustou, ppons, aubert\}@laas.fr
}

\begin{abstract}
For testing satellites before launching, multiple wired sensors are commonly used for covering the metallic walls of the inner satellite structure. In order to reduce the maintenance cost of such Structure Health Monitoring platform, wireless sensors could be advantageously used. In this paper, we demonstrate the reading feasibility of passive and wireless millimetre-wave sensors embedded inside an over-sized metallic box. The box simulates here a part of the electromagnetic reverberant environment within satellite structure. In the proposed crosspolarized configuration, a dual-polarized passive repeater is used for transmitting inside the box the co-polarized electric field radiated by a Frequency-Modulated Continuous-Wave $24 \mathrm{GHz}$ radar. This repeater is also used for transmitting outside the box the cross-polarized electric field radiated by a depolarizing sensor. The proof-of-concept is established here from a passive and chipless pressure sensor, but other types of wireless sensors may be used as well.

Keywords - wireless sensors, passive repeater, millimetrewave, electromagnetic reverberant environment, multiple reflections, multipath, field depolarization.
\end{abstract}

\section{INTRODUCTION}

Platforms dedicated to the testing of satellites subjected vibrations and shocks use traditionally wired sensors [1]. However, the maintenance cost of such sensors is high, and the risk of wired connection breaks is critical. To overcome such issues, wireless sensors could replace wired sensors.

As the inner structure of satellites is typically a closed electromagnetic reverberant environment (see Fig. 1), the wireless reading from the outside of sensors embedded inside satellites is very challenging. However, as indicated in Fig. 1, the outer metallic walls of satellites may present small apertures or slots, which could be advantageously used for transmitting an electromagnetic wave inside the satellite. However, such small openings may not transmit enough electromagnetic power inside the structure for ensuring the reliable wireless communication between the reader and embedded sensors. The first idea proposed here is to guarantee the wireless link by using passive repeaters (see, e.g., [2]): the electromagnetic field radiated by the reader would then be received by the Rx-antenna of the repeaters, and then transmitted inside the satellite by using a short transmission line passing through available apertures or slots. However, multiple electromagnetic reflections inside the metallic structure of the satellite may render impractical the wireless link between the reader and embedded sensors. To solve this issue, the second idea consists of using both co- and crosspolarized electric fields in order to mitigate the undesirable effect of reflections and multipaths.

In this paper, we demonstrate that passive millimetre-wave sensors embedded inside an over-sized metallic box can be wirelessly interrogated. The box simulates here a part of the electromagnetic reverberant environment within a typical satellite structure. A dual-polarized passive repeater is used for transmitting inside the box the co-polarized electric field radiated by a FM-CW (Frequency-Modulated ContinuousWave) $24 \mathrm{GHz}$ radar. This repeater is also used for transmitting outside the box the cross-polarized electric field radiated by a depolarizing sensor.

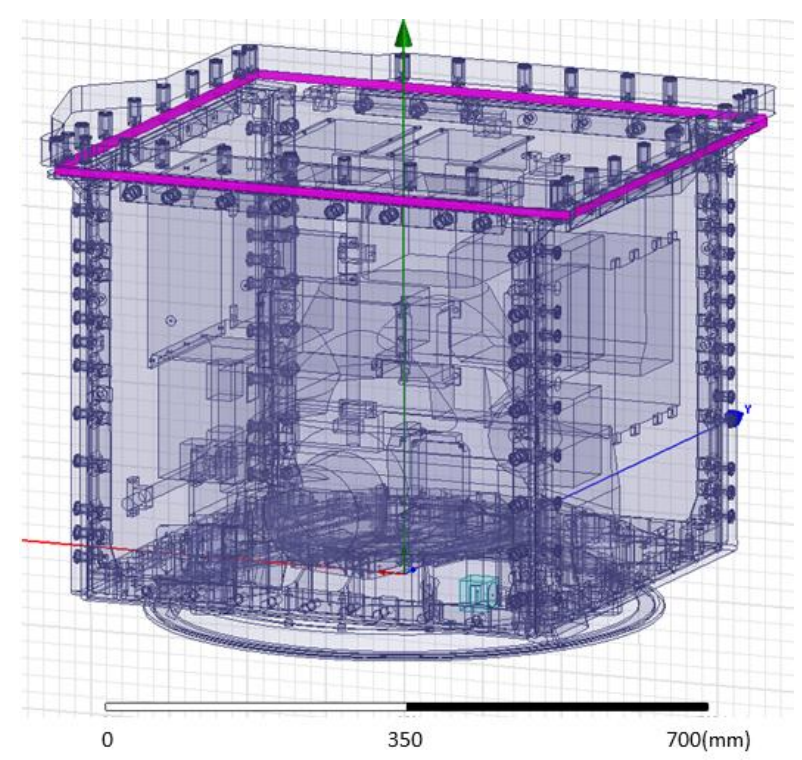

Fig. 1. Computer-aided design of a typical structure of a telecommunication satellite (courtesy of French Spatial Agency). Small available apertures in the outer wall of the satellite are indicated in pink at the top of the structure.

\section{EXPERIMENTAL SETUP}


The experimental setup is illustrated in Fig. 2. A cuboid metallic box $\left(0.60 \times 0.32 \times 0.35 \mathrm{~m}^{3}\right)$ perforated by a small hole has been manufactured for simulating one of the multiple metallic cavities located behind the outer walls of satellites. The hole simulates a small aperture, which may eventually exist on the satellite walls. All antennas are horn antennas of gain $20 \mathrm{dBi}$ and operating at $23.8 \mathrm{GHz}$.

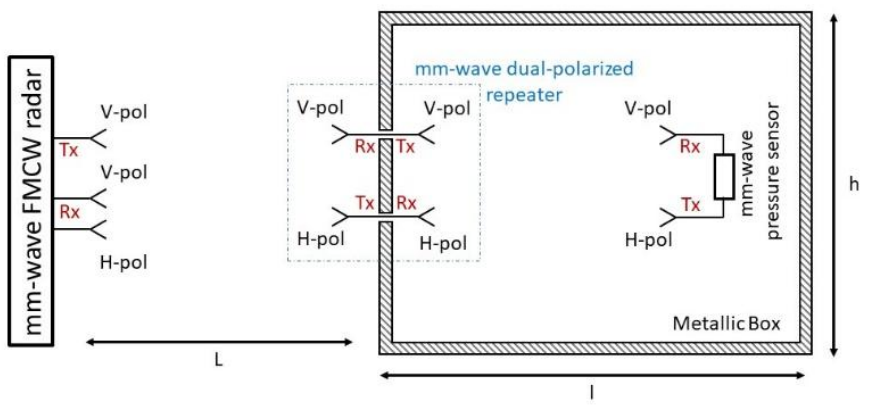

(a)

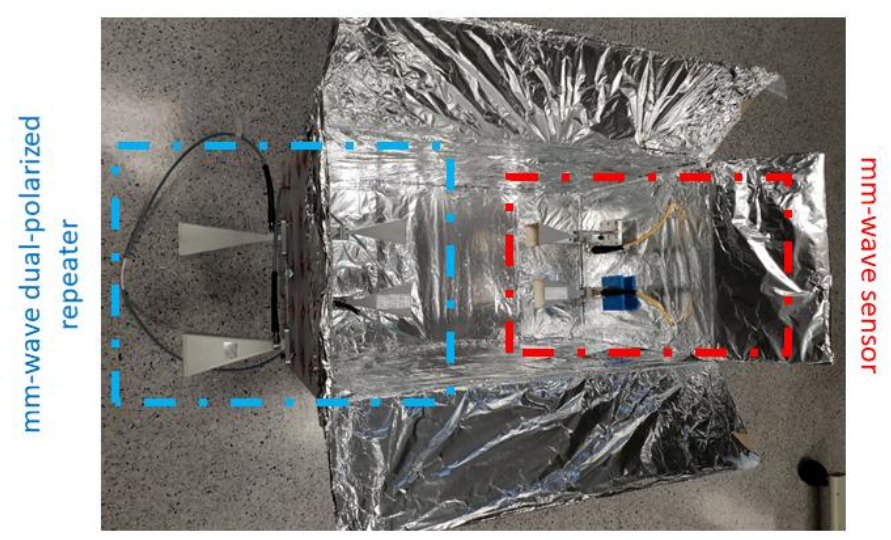

(b)

Fig. 2. (a) Experimental setup including the $24 \mathrm{GHz}$ FM-CW radar (radar-towall separation distance $\mathrm{L}=0.8 \mathrm{~m}$ ), the dual-polarized through-wall passive repeater (wall thickness $=0.02 \mathrm{~mm}$ ), the oversized metallic box $(0.60 \mathrm{~m} \mathrm{x}$ $0.32 \mathrm{~m} \times 0.35 \mathrm{~m})$ and the $\mathrm{mm}$-wave passive and wireless pressure sensor $(1=$ $0.5 \mathrm{~m})$; (b) Top view of the opened metallic box. Inside the box are located the passive sensor and the through-wall repeater.

To perform the remote reading of the passive sensor enclosed in the metallic box, a frequency-modulated (FM) electric field (E-field) is radiated by the vertically polarized (Vpolarized) Tx-antenna of a FM-CW radar operating at $23.8 \mathrm{GHz}$ (bandwidth of $2 \mathrm{GHz}$ ). The E-field is received by the Vpolarized Rx-antenna of the passive repeater, and transmitted inside the box by using a coaxial line $(1 \mathrm{~m})$ passing through the hole of the box. The V-polarized Tx-antenna of the repeater reradiates inside the box the FM E-field, which is then received by the V-polarized Rx-antenna of the passive pressure sensor.

The transmission loss in the sensor depends here on the pressure at sensor location. As a matter of fact, the pressure sensor in our present study consists of a silicon membrane and a half-wavelength parallel-edge coupled-line microstrip resonator operating in the radar frequency bandwidth: when the pressure applied on the membrane changes, a deformation of the membrane occurs and modifies the propagation loss (see
[3] and Section IV). As a result, the magnitude of the electric field re-radiated by the H-polarized Tx-antenna of the sensor depends on the applied pressure. This E-field is finally through-wall transmitted outside the metallic box thanks to the passive dual-polarized repeater, and it is received by the $\mathrm{H}$ polarized Rx-antenna of the radar. From the Fast Fourier Transform of the product between the received signal and FM signal transmitted by the radar, the beat frequency spectrum is obtained and, the amplitude of the radar echo (or echo level) is then derived as a function of the separation distance from the radar (see, e.g., [4] for the detailed derivation of radar echoes).

\section{COMPARATIVE STUDY BETWEEN CO-POLARIZED AND CROSS-POLARIZED CONFIGURATIONS}

The experimental wireless set up described in section II uses both co- and cross-polarized electric fields. This configuration is called here the cross-polarized configuration. The setup can also use only V-polarized (resp., only $\mathrm{H}$ polarized) E-fields from rotating by $90^{\circ}$ all H-polarized (resp., all V-polarized) horn antennas. The co-polarized configuration designates here the experimental setup for which all antennas (i.e., Tx- and Rx-antennas of the radar, repeater and sensor) are V-polarized. To highlight the advantages of the cross-polarized configuration, the two following experiments are performed.

\section{A. Experiment $n^{\circ} 1$}

The mm-wave sensor shown in Fig. 2 is first replaced by a series short-circuit or a series open-circuit. The series shortcircuit simulates low transmission losses through the sensor and allows re-radiating the received electric field. At the opposite, no transmission (and consequently, no re-radiation) occurs when the series open-circuit replaces the sensor. Fig. 3 reports the difference between the echo level measured for the series short-circuit and one measured for the series opencircuit. This difference is displayed as a function of the separation distance from the radar both for the cross- and copolarized configurations. Contrary to the co-polarized configuration, the cross-polarized configuration allows observing a large echo level difference $(20 \mathrm{~dB})$ between the short and open-circuit conditions. This experimental result can be interpreted as follows:

$i$. For the co-polarized configuration, the E-field is transmitted inside the cuboid metallic box, and multiple reflections generate a co-polarized E-field of high magnitude, but a crosspolarized E-field of low magnitude.

ii. For the cross-polarized configuration, the H-polarized Txantenna of the two-port network radiates the cross-polarized electric field, which brings the information on the loading condition (open or short-circuit). Consequently, the echo level difference between the short- and open-circuit conditions is large.

The cross-polarized configuration should be then preferred to the co-polarization configuration for the remote detection of loading condition (open- or short-circuit). In Fig. 3, delayed peaks are measured in the difference between the two echoes 
for the cross-polarized configuration. They are due to the multiple reflections inside the box. As a matter of fact, these late peaks are created by the structural scattering mode of the sensor antennas, which is reflected by the metallic wall in front of these antennas, and is finally received and re-radiates by the sensor. The separation distance $(63 \mathrm{~cm})$ between two successive peaks is actually close to the length of one roundtrip from the sensor antenna to the front metallic wall.

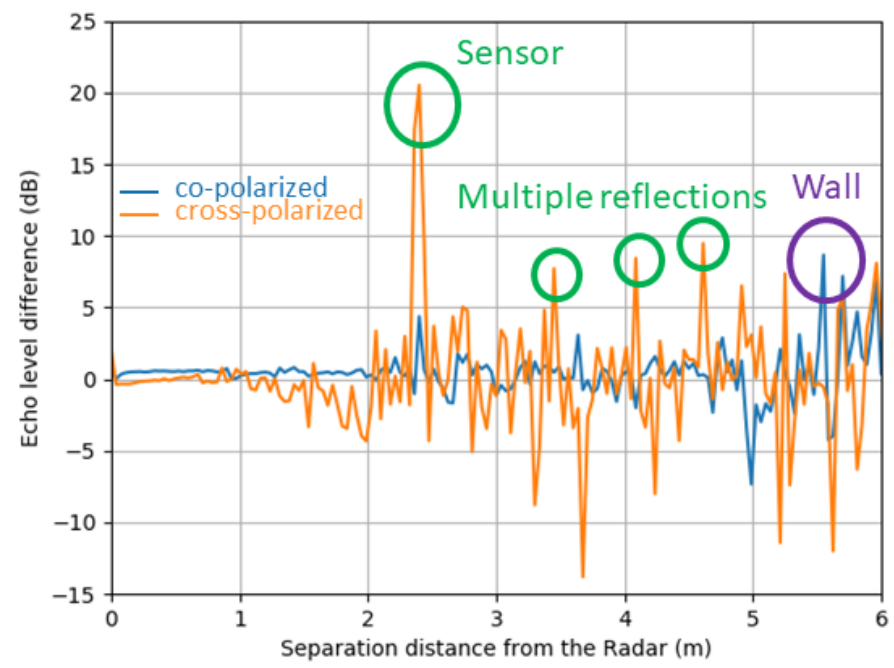

Fig. 3. Difference between two echo levels: one is obtained when the sensor is replaced by a series short-circuit, and the second is obtained when the sensor is replaced by a series open-circuit (Experiment $\left.n^{\circ} 1\right)$. The difference is given as a function of the distance from the radar, and for the cross-polarized (in orange) and co-polarized (in blue) configurations.

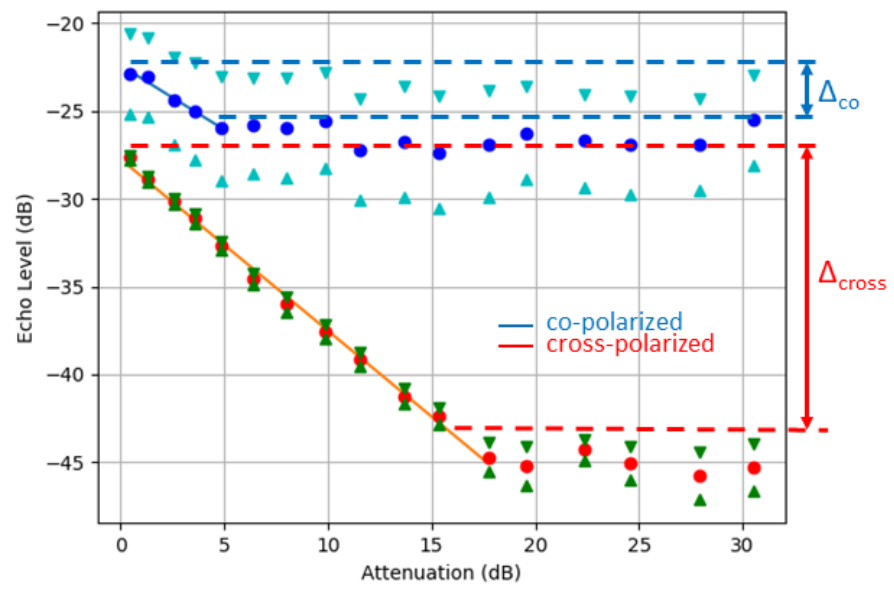

Fig. 4. Measured radar echo level as a function of the propagation loss (or attenuation) in the two-port attenuator in the cross-polarized configuration (red) and in co-polarized configuration (blue). The mean value (point) and standard deviation (error bars) of echo levels are computed here from 100 measured echoes.

\section{B. Experiment $n^{\circ} 2$}

The mm-wave sensor shown in Fig. 2 is now replaced by an adjustable attenuator $(0-30 \mathrm{~dB})$. For a given attenuation (or propagation loss), the mean value and standard deviation of the echo level are computed from 100 measured echoes. As shown in Fig. 4, for attenuation between $0 \mathrm{~dB}$ and $17 \mathrm{~dB}$ and in the cross-polarized configuration, the echo level depends linearly on the attenuation. The coefficient of determination $\mathrm{R}^{2}$ of the linear regression is of 0.99 and the slope of the linear regression is very close to 1 . The full-scale range of the echo level, denoted $\Delta_{\text {cross, }}$ is found to be of $17 \mathrm{~dB}$. This full-scale range, denoted $\Delta_{\mathrm{co}}$ in the co-polarized configuration, is significantly lower $\left(\Delta_{\mathrm{co}}=4 \mathrm{~dB}\right.$, with $\left.\mathrm{R}^{2}=0.91\right)$. Moreover, the standard deviation of the echo level is higher $(3.1 \mathrm{~dB}$ in the linear region) in the co-polarized configuration than the one provided by the cross-polarized configuration $(1 \mathrm{~dB}$ in the linear region). As physically highlighted in section III.A, a weak cross-polarized electric field is generated by the cuboid metallic box when the electric field undergoes multiple reflections. Consequently, when the cross-polarized configuration is used, only the sensor radiates the crosspolarized electric field from which information on the propagation loss provided by the attenuator can be derived.

\section{WIRELESS READING OF EMBEDDED MM-WAVE SENSOR}

In this section, a millimeter-wave and passive pressure sensor is wirelessly interrogated in the cross-polarized configuration illustrated in Fig. 2.

The sensor is a silicon membrane and a half-wavelength parallel-edge coupled-line microstrip resonator [3]. It is manufactured on a $500 \mu \mathrm{m}$ thick borosilicate glass (B33) substrate. A $0.05 \mu \mathrm{m}$ thick titanium (Ti) and a $0.5 \mu \mathrm{m}$ thick copper $(\mathrm{Cu})$ layers are first deposited. The resonator ports thickness is then increased until $9 \mu \mathrm{m}$ through a $\mathrm{Cu}$ electrochemical growth. The protection of the structure against oxidation due to further high-temperature processes is ensured by a thin electroless deposited gold ( $\mathrm{Au}$ ) layer. The resonator is located inside a $5.5 \mathrm{~mm}$ diameter cylindrical cavity formed by a $10 \mu \mathrm{m}$ thick spincoated low-losses RF photoresist, on which a $100 \mu \mathrm{m}$ thick high resistivity silicon ( $\mathrm{Si}-\mathrm{HR}$ ) membrane is bonded at $100^{\circ} \mathrm{C}$. The ground plane at the bottom face is composed of a $0.05 \mu \mathrm{m}$ thick layer of chromium $(\mathrm{Cr})$ and of a $1.5 \mu \mathrm{m}$ thick layer of aluminum (Al) (see Fig. 5(a)). Finally, the microwave transducer is placed inside a $11.25 \times 8.3 \times 5.56 \mathrm{~mm}^{3}$ Al metallic cavity (Fig. 5(b)).

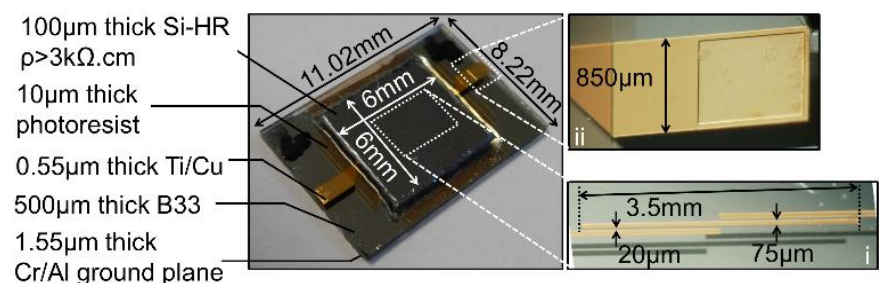

(a)

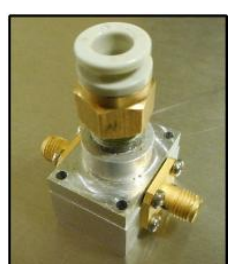

(b) 
Fig. 5. (a) Views of the mm-wave pressure sensors with its Si-HR membrane; (b) Views of sensor package (dimensions are of $3.6 \times 2.4 \times 4.2 \mathrm{~cm}^{3}$ (see [3] for more details).

The transmission loss in the mm-wave sensor depends on the pressure at sensor location. The measured transmission loss (or attenuation) in the radar operating bandwidth for an applied pressure ranging from 0 to $2.5 \mathrm{bar}$ is displayed in Fig. 6 .

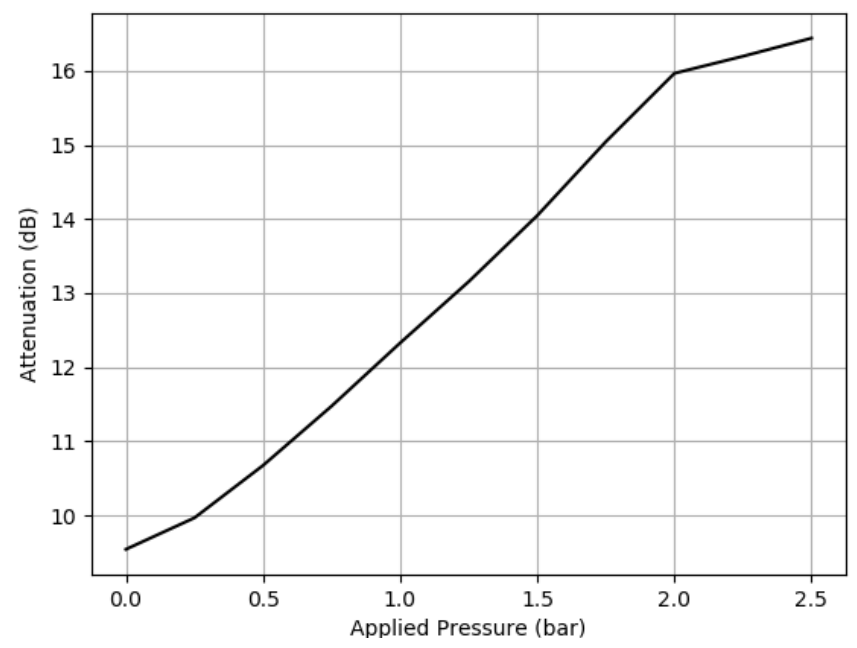

Fig. 6. Measured attenuation on the field propagating through the two-port sensor at between $23 \mathrm{GHz}$ and $25 \mathrm{GHz}$ for applied pressure ranging from 0 to 2.5 bar.

For the reasons given in Section III, the cross-polarized configuration is adopted here for the remote derivation of the applied pressure. Fig. 7 reports the variation of the echo level as a function of the applied pressure on the sensor membrane.

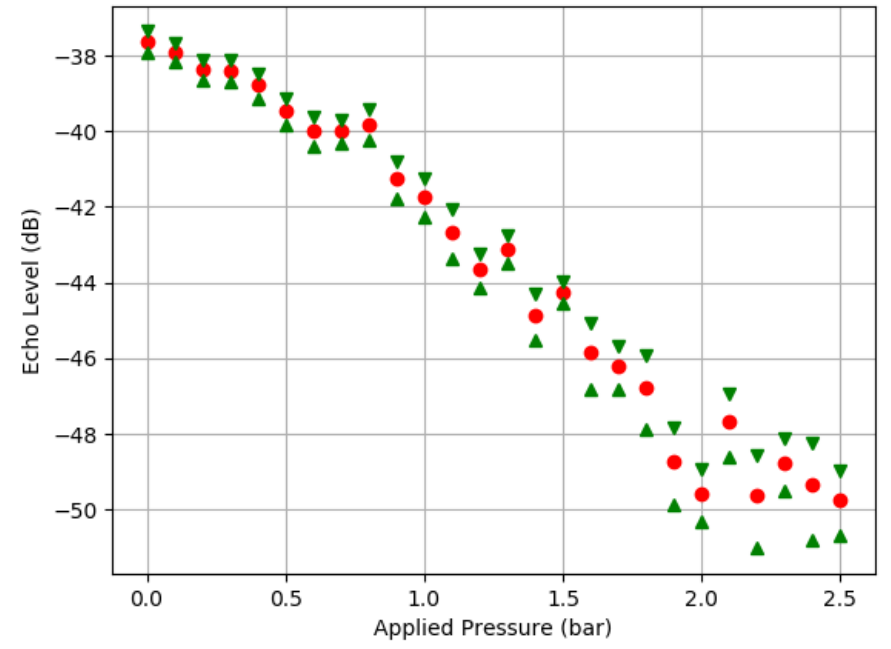

Fig. 7. Measured radar echo level as a function of the pressure applied on the sensor membrane. These measurement results have been obtained from the cross-polarized configuration. The mean value (red point) and standard deviation (or error bars, green symbols) of echo levels are computed here from 100 measured echoes.

The available full-scale pressure range is of 2.5 bars. As the measurement uncertainty on the echo level does not exceed $1.1 \mathrm{~dB}$ for higher applied pressure $(>2.2 \mathrm{bar})$, the measurement uncertainty on the pressure is of 0.4 bar. The measurement accuracy on lower applied pressures $(<0.2$ bar $)$ is higher, as the standard deviation on the echo level does not exceed 0.2 bar. Significantly worse results (not shown here) were obtained by using the co-polarized configuration. Due to multiple reflections, this configuration does not allow the remote detection of the pressure sensor place inside the metallic box. But, the use of cross-polarized configuration and dual-polarized repeater allows demonstrating the feasibility of the wireless reading of millimetre-wave passive sensors embedded metallic box. The reason is that the multiple reflections in the cuboid metallic box generate a weak contribution to the cross-polarized electric field re-radiated by the sensor.

\section{CONCLUSION}

In order to wirelessly interrogate the passive millimetre-wave pressure sensor embedded inside a metallic box, which simulates a part of the satellite inner structure, we have proposed to use a dual-polarized passive repeater. It allows transmitting inside the box the co-polarized electric field radiated by the FM-CW radar reader, and transmitting outside the box the cross-polarized electric field radiated by the depolarizing sensor. The proof-of-concept has been established by using a pressure sensor, but strain wireless gauges, such as those reported in [5], could be used as well.

Further works include the miniaturization of the repeater by using, e.g., planar antennas instead of bulky horn antennas, and to cascade dual-polarized repeaters inside the satellite in order to create multiple wireless channels inside the satellite and reading sensors distributed inside the overall satellite structure.

\section{ACKNOWLEDGMENTS}

The authors wish to acknowledge the French Space Agency (CNES) for financial support, and Leny Baczkowski and Bertrand Marty (CNES) for helpful technical information about satellite structure and testing.

\section{REFERENCES}

[1] P.-E. Dupuis, G. Pélissier, F. Deneuve, "Sensor Network Development", European Conference on Spacecraft Structures, Materials \& Environmental Testing, Toulouse, France, 27-30 September 2016.

[2] A. Ali, A. Coustou, H. Aubert, "Wideband Passive repeaters: Design and Measurements," IEEE Antennas and Propagation Society International Symposium, Charleston, South Carolina, USA, 1-5 June 2009.

[3] J. Philippe, D. Henry, M. V. De Paolis, A. Rumeau, A. Coustou, P. Pons, H. Aubert, "Wireless Remote Monitoring of Packaged Passive Sensor for In-situ Pressure Measurement in Highly Reflective Environments," IEEE International Microwave Symposium, Philadelphia, Pennsylvania, USA, 10-15 June 2018.

[4] B. A. Atayants, V. M. Davydochkin, V. V. Ezerskiy, V. S. Parshin, and S. M. Smolskiy, Precision FMCW Short-Range Radar for Industrial Applications. Norwood, MA, USA: Artech House, 2014. 
[5] T.Thai, H.Aubert, P.Pons, G.DeJean, M.-M.Tentzeris, R. Plana, "Novel Design of a Highly Sensitive RF Strain Transducer for Passive and Remote Sensing in Two Dimensions," IEEE Transactions on Microwave Theory and Techniques, Vol. 61, Issue 3, pp. 1385-1396, March 2013. 